\title{
Optimization of Cluster Heads Selection by Imperialist Competitive Algorithm in Wireless Sensor Networks
}

\author{
Marjan Maadi \\ Department of IT engineering \\ Graduate university of advanced Technology \\ Kerman, Iran
}

\author{
Mansoureh Maadi \\ Department of Industrial Engineering \\ Damghan University \\ Damghan, Iran
}

\begin{abstract}
Wireless Sensor Networks are one of the most important distributed networks which are used in the wide range of applications. Energy is one of the major limitations of wireless sensor networks, which has direct impact on the network's life time. LEACH protocol is one of the most wellknown Clustering schemes that select cluster heads randomly. Clustering is an effective topology control Approach in wireless sensor networks. In this paper, we proposes new clustering algorithm that using an imperialist competitive algorithm to select cluster heads in LEACH algorithm. Simulation results show proposed algorithm can prolong the network lifetime efficiently compared with LEACH protocol.
\end{abstract}

\section{General Terms}

Meta heuristic algorithms, clustering.

\section{Keywords}

Imperialist competitive algorithm, clustering, wireless sensor networks, energy consumption.

\section{INTRODUCTION}

In the recent years, Wireless Sensor Networks have witnessed many advances which lead to wide range of applications. Wireless Sensor Networks are constructed using tiny sensor nodes. These sensor nodes can sense and collect data from the environment and send them to the Base Station via wireless channels. Each node works independently and has some limitations like energy resource, computational power and memory size [1]. As a consequence of these constrains, Wireless Sensor Networks have their own challenges, therefore most of the traditional protocols do not apply on them. These challenges guide researchers to plenty of new research areas. Wireless Sensor Networks are used in wide range of areas like military, home, healthcare applications etc. In all of these applications, energy limitation is a very important factor. The nodes energy depletes the functionality of the network impairs. Besides, when the network loses many nodes, the network lifetime will be ceased so the dead nodes should be replaced. But in the most of applications like military environment, it is impossible to replace the nodes. As a result, considering these constrains in the proposed protocol is essential.

Regarding the limitations of Wireless Sensor Networks, designing energy-aware algorithms is an important factor in order to increase the network lifetime[2]. One of the methods to improve energy consumption in Wireless Sensor Networks is using topology management schemes [3]. These schemes consist of three steps: topology discovery, sleep round management and clustering.
Clustering is a process in which the nodes of network are divided into groups called clusters. In each cluster, a node is selected as cluster head. In the most of the schemes, the nodes send their data to the cluster heads directly, then the cluster heads aggregate the collected data and send them to the Base Station. LEACH is the first and the most popular clustering protocol [4]. One of the major problems in LEACH clustering is choosing the cluster head which is done randomly. this can lead to unbalanced clusters which decreases the network efficiency.

In recent years, a lot of work has been done to improve LEACH protocol. Loscri et al. [5] proposed Two-Levels Hierarchy for Low-Energy Adaptive Clustering Hierarchy (TL-LEACH).This algorithm work better in avoiding large energy loss in the long distance transmission. Tabibzadeh et al. [6] proposed Chain-based LEACH that improved to significantly reduce energy consumption by merging LEACH and PEGASIS algorithm [7]. Handy et al. [8] proposed an improved algorithm called Low Energy Adaptive Clustering Hierarchy with Deterministic Cluster head Selection (LEACH-DCHS) which takes the residual energy information into account and can elect deterministically those nodes with more remaining energy as cluster head.

The rest of this paper is organized as follows: Section 2 describes LEACH clustering. Section 3 explain Imperialist competitive algorithm, Proposed ICA-LEACH clustering algorithm are described in section 4. Simulation and results are presented in Section 5 and finally section 6 is conclusion of paper.

\section{LEACH}

As said before, one of the main clustering approaches is LEACH protocol. In this protocol, time is divided into equal parts and each part is called a round. Also each round includes two steps: Initializing phase and Stability phase. In the first phase, clusters are formed and in the second phase network continues to perform its normal activities, like collecting and transferring data [4]. At first, every node i generates a uniform random number in the interval $[0,1]$ at the beginning of each round, if this number is less than the $\mathrm{T}(\mathrm{n})$ probability function, then this node elects itself to be a cluster head. The T(n) function calculated using below equation.

$$
\left\{\begin{array}{lr}
\frac{P}{1-P *\left(r \bmod \frac{1}{p}\right)} & \text { if } n \in G \\
0 & \text { otherwise }
\end{array}\right.
$$

Where $\mathrm{G}$ is set of nodes that have not been cluster head in previous $1 / \mathrm{p}$ rounds, $\mathrm{P}$ is the desired percentage of cluster heads, $\mathrm{r}$ is current round. The node becomes cluster head in current round. It will be cluster head after next $1 / p$ 
rounds. This indicates that every node will serve as a cluster head equally and energy consumption will be distributed evenly among nodes. Elected cluster head broadcasts its status using CSMA MAC protocol. Based on the received signal strength, the non-cluster nodes will determine to join which cluster, then the nodes send message to cluster heads. Based on numbers of nodes in a cluster, cluster head schedule a TDMA, and specifies communication time interval for each node. By receiving the TDMA schedule, nodes communicate to cluster head during their allocated time slots, otherwise nodes keep sleeping. When data from all nodes have been received by the cluster heads, they will aggregate, compress and transmit them to base station.

\section{IMPERIALIST COMPETITIVE \\ ALGORITHM}

Imperialist competitive algorithm (ICA) that was proposed by Atashpaz and Lucas (2008), is an evolutionary algorithm that inspired by socio-political process of imperialistic competition. This algorithm shows good capability in diverse optimization tasks [9]. Like other evolutionary algorithms, it starts with an initial population which is called country. Some of the best countries are chosen as imperialist and the rest are the colonies of these imperialists. After that, all the colonies of the initial population are distributed among imperialists based on imperialist's powers. The more powerful imperialists have the more colonies. An imperialist and its colonies create an empire. After construction initial empires, in each empire, the imperialist tries to assimilate its colonies and move them toward itself. After that, Imperialistic competition among these empires forms the proposed evolutionary algorithm. In the competition the power of each empire is related to the power of imperialist and the percentage of the mean power of its colonies. During this competition, any empire which is not strong enough and cannot prevent decreasing its power will be eliminated. Weak empires collapse and powerful ones take possession of their colonies. The movement of colonies toward their relevant imperialists (assimilation policy) and completion Imperialistic competition cause algorithm converges to a state in which there exists only one empire that is the optimal solution of the problem. In this ideal new world colonies have the same position and power as the imperialist. The flowchart of ICA algorithm is shown in figure 1 .

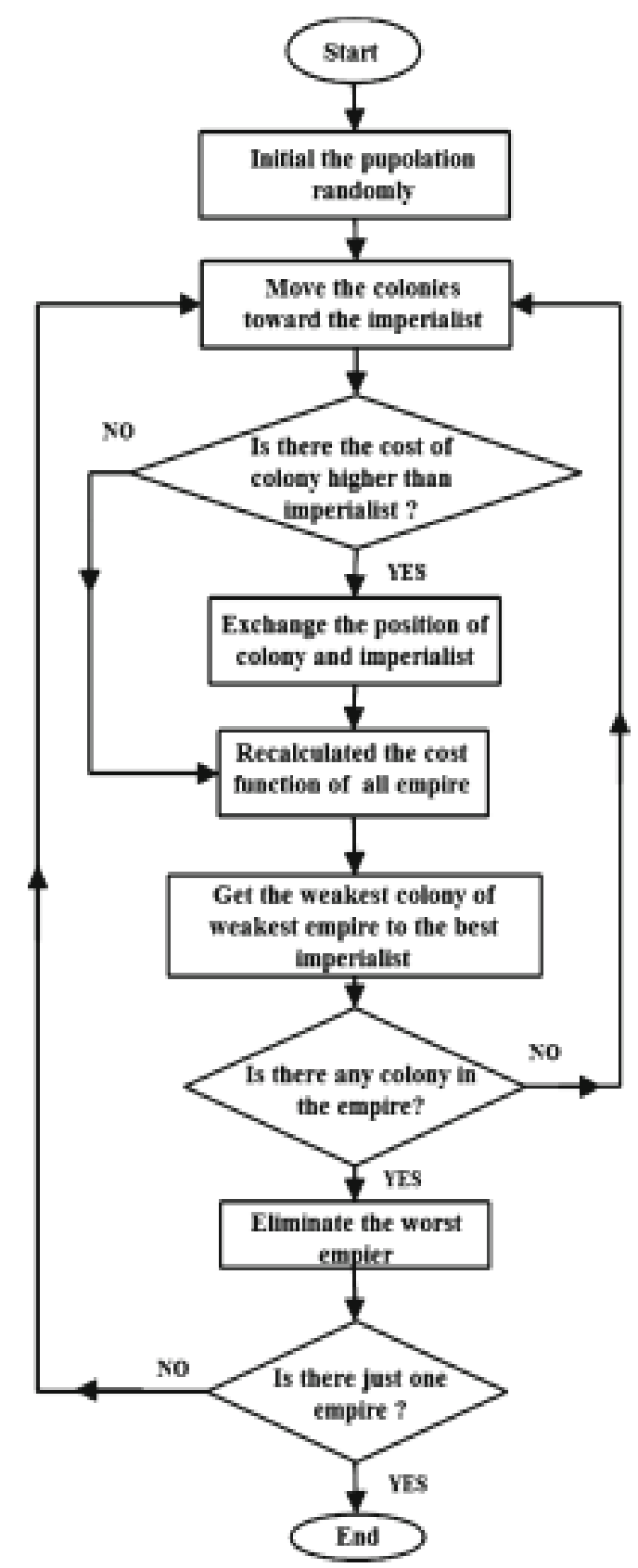

Fig. 1: ICA flowchart

\section{ICA_LEACH PROPOSED ALGORITHM}

As mentioned before, in proposed algorithm, instead of cluster head random selection in LEACH clustering algorithm, we use metaheuristic imperialist competitive algorithm to select appropriate cluster heads to increase network lifetime and network efficiency. Indeed, in each round of LEACH algorithm in proposed algorithm, optimized cluster heads are selected by imperialist competitive algorithm. Figure 2 shows the flowchart of proposed algorithm. 


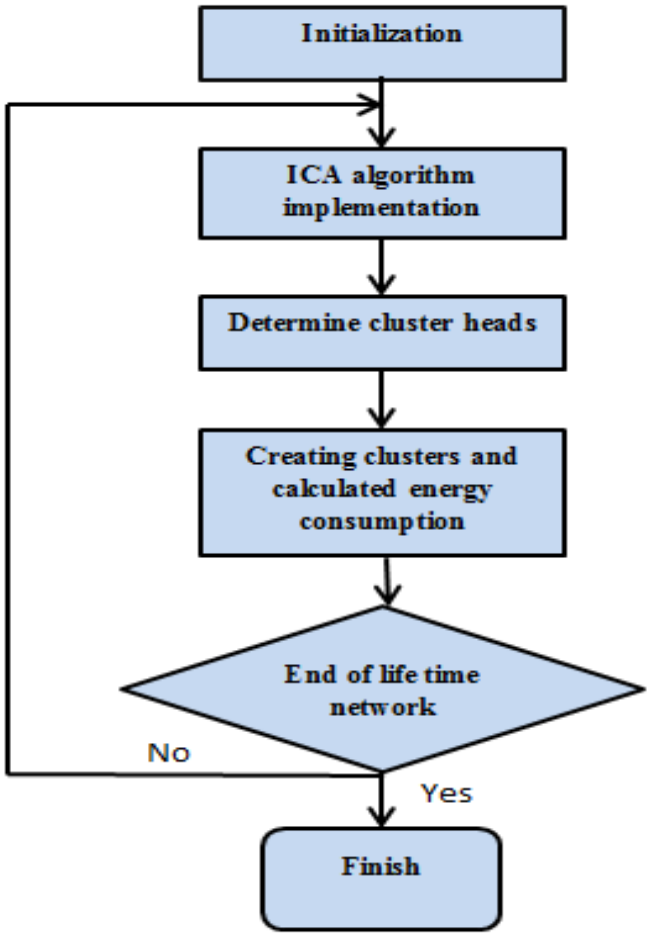

Fig. 2: flowchart of proposed algorithm

According to the flowchart, after setup the initial network randomly, ICA-LEACH proposed algorithm uses ICA algorithm in cluster heads selection, then, LEACH algorithm is implemented. To summarize the steps of proposed algorithm, In following sections steps of ICA algorithm to select appropriate cluster heads is described and steps of leach algorithm is done according to leach algorithm which is declared in section 2.Thus, In proposed algorithm, at first a random initial network is created and with this network, ICA starts its steps as follows.

\subsection{Generating initial empires}

ICA starts with some initial population named country which is an array of variables that should be optimized. Indeed, a country is a feasible solution of the optimization problem. In proposed algorithm after setting up a random network, a country is defined as a $1 \times n$ vector of binary numbers which $n$ is the number of network's nodes. Indeed a country is a network with ordinary and cluster head nodes. Each bit of the vector shows a node of the network that if the node is an ordinary node the related bit is zero otherwise if a node is a cluster head, the bit is one. Figure 3 shows a random country. As you see, this country shows a network with 10 nodes that nodes 1,4 and 8 are cluster heads and others are ordinary nodes. Another country for this network can be a $1 \times n$ vector of binary numbers which nodes 2, 4 and 9 are cluster heads and others are ordinary nodes. It is noticeable that the number of cluster heads in a network is 3 to 5 percent of nodes of network [10].

\begin{tabular}{|l|l|l|l|l|l|l|l|l|l|}
\hline 1 & 0 & 0 & 1 & 0 & 0 & 0 & 1 & 0 & 0 \\
\hline
\end{tabular}

Fig 3: A country
After generating initial countries randomly, since each country shows a network with different cluster heads, cost function is defined as the energy consumption of network for each country. After consuming cost function for all countries, the most powerful countries (countries with minimum cost function) are selected as imperialist and other countries are colonies of these imperialists. To proportionally divide the colonies among imperialist we define the normalized cost of an imperialist as follow:

$$
N C_{j}=\max _{i}\left\{c_{i}\right\}-c_{j} \quad i, j=1,2, \ldots, N_{\text {imp }}
$$

Where $\mathrm{C}_{\mathrm{j}}$ is the cost of $\mathrm{jth}$ imperialist and $\mathrm{Nc}_{\mathrm{j}}$ is its normalized cost. Having the normalized cost of all imperialists and Because of inverse relationship between cost and power, the normalized power of each imperialist is defined as follow:

$p_{j}=\left|\frac{N C_{j}}{\sum_{i=1}^{N i m p} N C_{i}}\right| \quad j=1,2, \ldots, N_{\text {imp }}$

The power of each imperialist can be the number of its colonies. The more powerful an imperialist is, the more colonies it has. Thus the initial number of colonies of an imperialist is computed as follow:

$$
\operatorname{Nocol}_{j}=\operatorname{round}\left\{p_{j} \cdot N_{\text {col }}\right\} \quad \mathrm{j}=1,2, \ldots, \mathrm{N}_{\mathrm{imp}}
$$

Where Nocol $_{j}$ is the initial number of colonies of $j$ th imperialist. But the question is which colonies belong to which imperialists. In ICA, distribution of colonies among imperialists is done randomly. Thus we randomly choose Nocol $_{j}$ colonies and give them to $j$ th imperialists. An imperialist and its colonies construct an empire. Figure (4) shows generating of initial empires. As shown in figure (4) the bigger imperialists have a greater number of colonies.

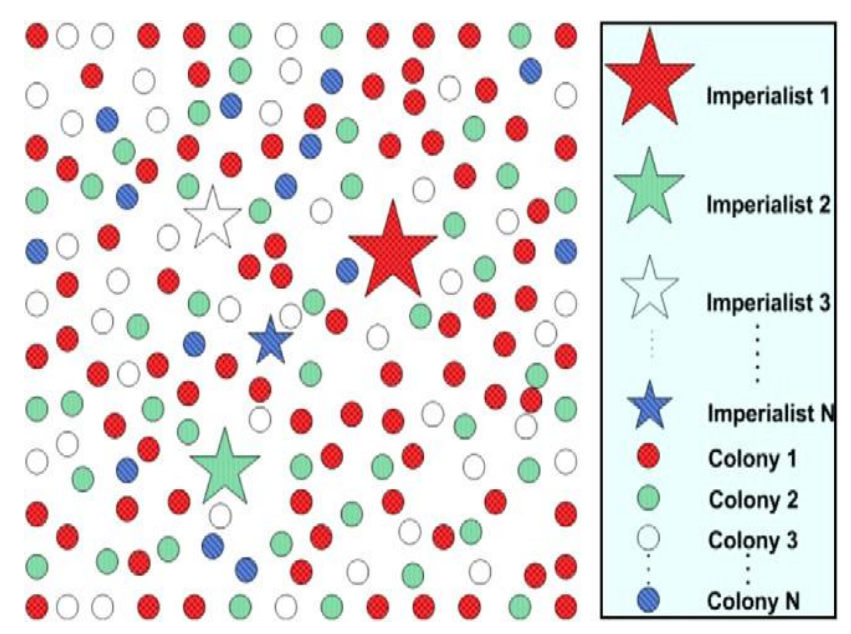

Fig. 4: Initial empires

\subsection{Assimilation Policy}

After generating initial empires, in every empire assimilation policy is done. Pursuing this policy, imperialists try to improve their colonies and start to absorb them. In ICA algorithm this policy is modeled by moving colonies toward their imperialist along different optimization axis. For example considering a 2- dimensional optimization problem figure 5 shows movement of a colony towards its relevant imperialist. In this figure $\mathrm{x}$ is a random variable with uniform 
(or any proper) distribution and shows the length of movement of colony toward its imperialist in the direction of the vector from the colony to the imperialist. Assimilating the colony by the imperialist didn't result in direct movement of the colonies toward the imperialist. To model this fact a random amount of deviation is added to the direction of movement. In figure 5, $\theta$ is a parameter with uniform (or any proper) distribution. Toward this movement some part of colony's structure will be similar to the imperialist structure.

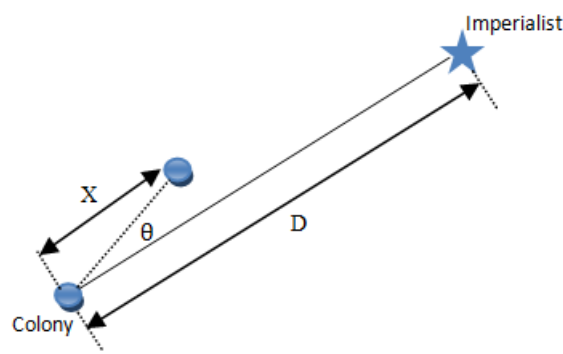

Fig. 5: assimilation policy

Since the goal of assimilation policy is to assimilate the structure of colony to its imperialist, In proposed algorithm we use some steps to implement this policy as follows:

- Consider a colony and its imperialist in an empire (Figure 6 )

- Determine the bits of cluster heads which are in the same place in imperialist and its colony.

- Determine $\mathrm{h}$ which is assimilation rate in [0 1].

- In imperialist country, for each bit 1(cluster head node) which is not 1 in the colony, select a random number such as $t$ in $\left[\begin{array}{ll}0 & 1\end{array}\right]$.

- If $\mathrm{t}<\mathrm{h}$ then the same node in colony country which is ordinary node changes to cluster head, otherwise investigate the next cluster head node in imperialist country. If in a colony an ordinary node changes to cluster head node, according to increase in the number of cluster heads in colony country, one of the cluster heads should changes to ordinary node. For this goal a cluster head in colony which has the least energy among cluster heads and in imperialist country is not a cluster head will be eliminated. this process will be continued till the last bit of imperialist will be investigated. One repetition of assimilation policy is shown in figure 7 .

- Compute the cost function of the colony in new position.

\begin{tabular}{|c|c|c|c|c|c|c|c|}
\hline Imperialist & $\underline{1}$ & 0 & 0 & 1 & 0 & 0 & 1 \\
\hline Colony & $\underline{1}$ & 0 & 1 & 0 & 0 & 1 & 0 \\
\hline
\end{tabular}

Fig 6: An imperialist and its colony

\begin{tabular}{|c|c|c|c|c|c|c|c|}
\hline Imperialist & $\underline{1}$ & 0 & 0 & 1 & 0 & 0 & 1 \\
\hline Colony & $\underline{1}$ & 0 & 1 & 0 & 0 & 0 & 1 \\
\hline
\end{tabular}

Fig 7: One repetition of assimilation policy

In proposed algorithm $\mathrm{h}$ is considered as 0.3 .

\subsection{Revolution}

In ICA Revolution approach is based on a fundamental change in power or organizational structures that takes place in a relatively short period of time. In the terminology of ICA, revolution causes a country to suddenly change its characteristics. Figure 8 shows the revolution in a colony.

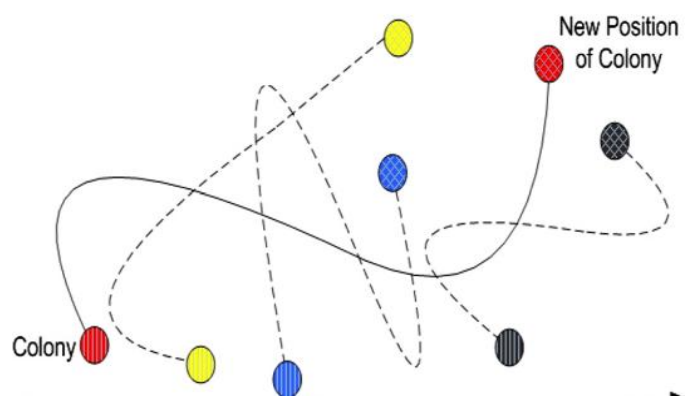

Fig 8: revolution

The revolution in colonies increases the exploration of the algorithm and prevents the early convergence of countries to local minimums. The revolution rate in the algorithm indicates the percentage of colonies in each empire which will randomly change their position. In proposed algorithm according to the revolution rate, in every empire a random colony is selected and one of the ordinary nodes of colony changes to a cluster head. After that, because the number of cluster heads in a colony increase, among other cluster heads of colony, a cluster head which has less energy changes to an ordinary node.

A very high value of revolution rate decreases the exploitation power of algorithm and can reduce its convergence rate. In our simulations the revolution rate is 0.3 that means 30 percent of colonies in each empire change their positions randomly.

\section{4 exchange positions of the imperialist and a colony}

While colonies move towards the imperialist, a colony might reach to a position with lower cost than the imperialist. In this case, the imperialist and the colony exchange their positions and the algorithm will continue by the imperialist in the new position and the colonies will be assimilated by the imperialist in its new position. Figure 9 depicts the position exchange between a colony and it's imperialist. In this figure the best colony of the empire is shown in a darker color. This colony has a lower cost than the imperialist. Figure 10 shows the empire after exchanging the position of the imperialist and its colony.

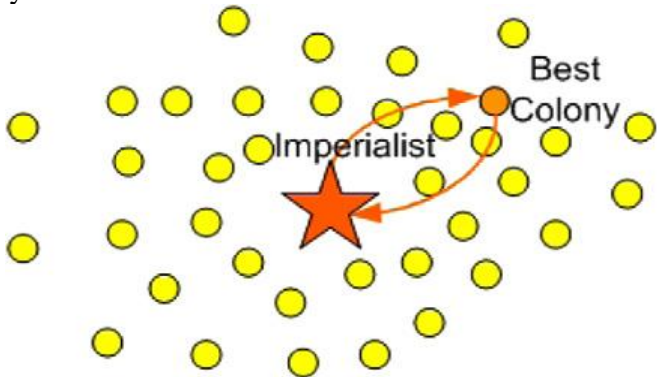

Fig 9: Exchanging the positions of a colony and its imperialist 


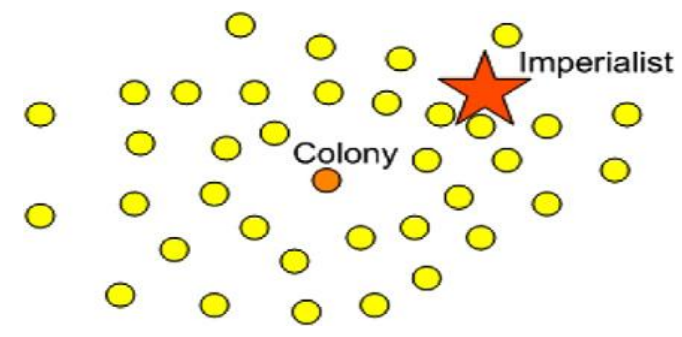

Fig. 10: The empire after position exchange

\subsection{Uniting Similar Empires}

In the movement of imperialists and colonies toward optimum solution, sometimes some imperialists might move to similar position. In such situation empires which their imperialists have similar position combine and construct one empire which in this empire one of the imperialists is imperialist of the new empire and colonies of empires are colonies of the new empire. With this empire algorithm continue. Figure 11 and figure 12 show the uniting process of two empires before and after uniting.

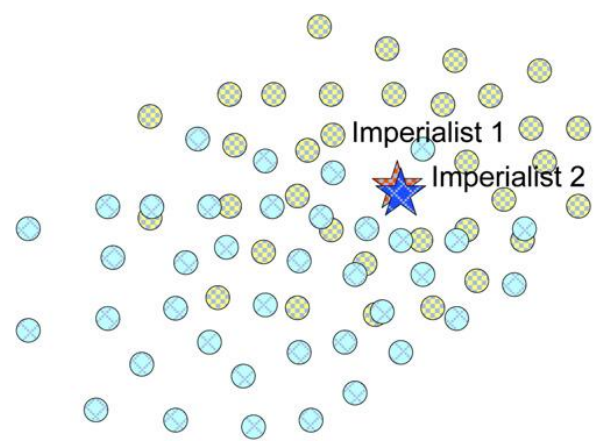

Fig. 11: Imperialists in two empire are in a similar position

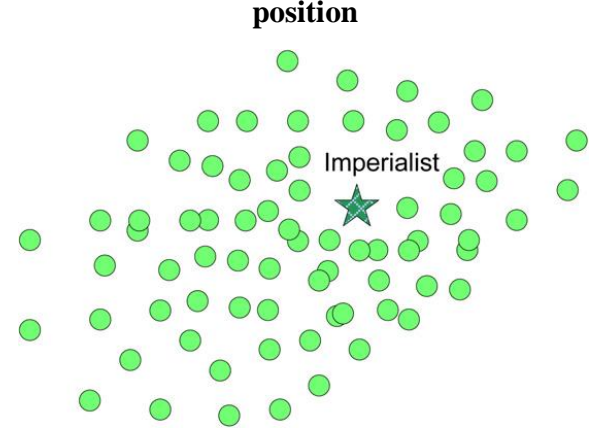

Fig. 12: Uniting Two Empires

\subsection{Imperialist Competition}

After assimilation policy and revolution in every empire, competition between empires starts. This competition is based on empires total power. Total power of an empire is affected by the power of imperialist and power of colonies of that imperialist. The total cost of an empire is computed as follow:

$\mathrm{TC}_{\mathrm{j}}=\mathrm{C}$ (imperialist) $+\xi$ mean (colonies of empires) ${ }_{\mathrm{j}}$

$\mathrm{j}=1,2, \ldots, \mathrm{N}_{\mathrm{imp}}$

Where $\mathrm{TC}_{\mathrm{j}}$ is the total cost of the empire and $\xi$ is a positive number which is considered to be less than 1. A little value for $\xi$ causes the total power of empire to be determined by just imperialist and colonies don't have effective role for determining power of empire, while increasing in $\xi$, increase the role of colonies in determining the power of an empire.

The value of $\xi=0.2$ has shown good results in this paper.

In imperialist competition all empires try to possess the other empire's colonies and control them. In this step, the power of weaker empires decrease and as a result the power of more powerful ones will increase. In ICA this competition modeled by just picking weakest colony of the weakest empire and making a competition among all empires to possess this colony. In this competition based on total power of empires, each empire will have a like hood of taking possession of the mentioned colony. In other words this colony will not be possessed by the most powerful empires; however powerful empires will be more likely to possess this colonies.

To start the competition, first the weakest colony of the weakest empire is chosen and then the possession probability of each empire should be computed based its total power. For computation possession probability of empires, first the normalized total costs of empires are calculated as follow:

$$
\mathrm{NTC}_{\mathrm{j}}=\max _{\mathrm{i}}\{\mathrm{Tc}\}_{\mathrm{i}}-\mathrm{Tc}_{\mathrm{j}} \quad \mathrm{i}, \mathrm{j}=1,2, \ldots, \mathrm{N}_{\mathrm{imp}}
$$

WhereTc $c_{j}$ and $\mathrm{NTc}_{\mathrm{j}}$ are total cost and normalized total cost of jth empire. Having normalized total cost, the possession probability of each empire is given by:

$$
W_{w_{j}}=\left|\frac{N_{T c}}{\sum_{i=1}^{N_{i m p}} N_{T c}}\right| \quad i, j=1,2, \ldots, N_{i m p}
$$

After computing possession probability for each empire, to divide the mentioned colonies among empires, vector $\mathrm{W}$ is formed as follow:

$$
\mathrm{W}=\left[\mathrm{w}_{\mathrm{w}_{1}}, \mathrm{w}_{\mathrm{w}_{2}}, \ldots, \mathrm{w}_{\mathrm{w}_{\mathrm{N}_{\mathrm{imp}}}}\right]
$$

Then a vector $\mathrm{R}$ with the same size as $\mathrm{W}$ whose elements are uniformly distributed random numbers is created,

$\mathrm{R}=\left[\mathrm{r}_{1}, \mathrm{r}_{2}, \ldots, \mathrm{r}_{\mathrm{N}_{\mathrm{imp}}}\right] \quad \mathrm{r}_{1}, \mathrm{r}_{2}, \ldots, \mathrm{r}_{\mathrm{N}_{\mathrm{imp}}} \sim \mathrm{U}(0,1)$

Then vector $\mathrm{D}$ is formed by subtracting $\mathrm{R}$ from $\mathrm{W}$ :

$$
\begin{aligned}
\mathrm{D}=\mathrm{W}-\mathrm{R}=\left[\mathrm{d}_{1}, \mathrm{~d}_{2}, \ldots, \mathrm{d}_{\mathrm{N}_{\mathrm{imp}}}\right]= \\
=\left[\mathrm{w}_{\mathrm{w}_{1}}-\mathrm{r}_{1}, \mathrm{w}_{\mathrm{w}_{2}}-\mathrm{r}_{2}, \ldots, \mathrm{w}_{\mathrm{w}_{\mathrm{N}_{\mathrm{imp}}}}\right. \\
\left.-\mathrm{r}_{\mathrm{N}_{\mathrm{imp}}}\right]
\end{aligned}
$$

Referring to vector $\mathrm{D}$, the mentioned colony is handed to an empire whose relevant index in D is maximized.

The modeled imperialist competition is depicted in figure 13 . In this competition the weakest colony of the empire1 (the weakest empire) is selected and other empires are competing for having that colony.

In the imperialist competition, the colonies of powerless empires will be distributed among other empires and empire will collapse. In modeling collapse mechanism different factors can be defined for a powerless empire. In proposed approach when an empire loses all its colonies it collapses.

After this step next round of ICA algorithm will start with assimilation policy. This process continues till stopping conditions occur. 


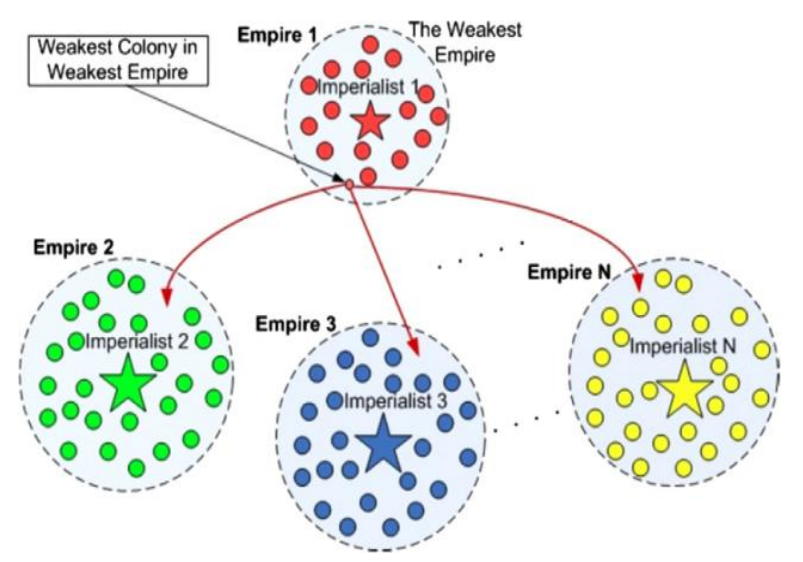

Fig. 13: Imperialist competition

\subsection{Stopping Condition}

Different criteria can be used to stop the algorithm. In proposed algorithm, the algorithm continues until $\mathrm{n}$ iteration is done or just one empire exists at end.

After implementation of ICA to select optimized cluster heads, proposed algorithm will be continued with next step of LEACH algorithm, which is collecting data of ordinary nodes by cluster heads in each cluster. After that cluster heads aggregate collective data and send them to base station. This process continues until the end of network life time.

\section{SIMULATION AND ANALYSIS}

The performance of the proposed protocol is evaluated using several tests. For the simulation, we have considered a scenario in which 100 nodes are distributed in the area randomly. The nodes are distributed in the $100 * 100$ environment.

Number of alive nodes in 1300 simulation rounds is shown in figure 14. As can be seen, the death rate of nodes in LEACH algorithm is faster than LEACH-ICA algorithm. This is due to the appropriate selection of cluster heads using the meta heuristic ICA algorithm.

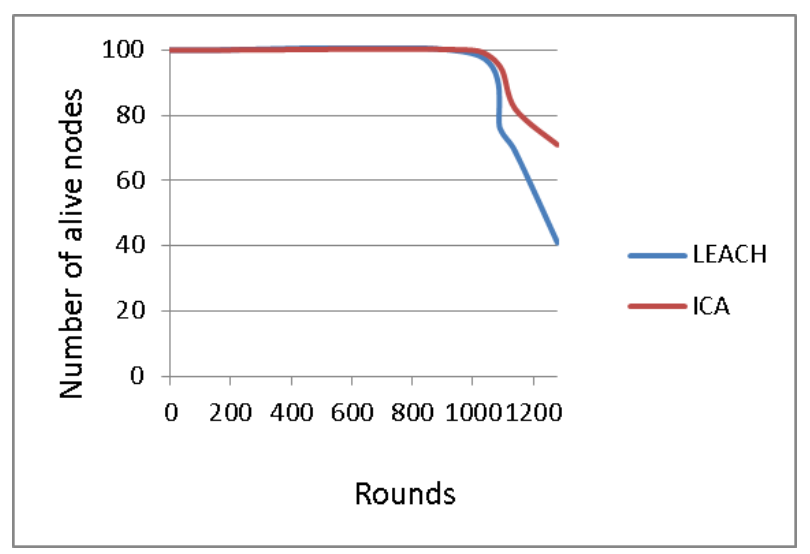

Fig. 14: comparison of alive nodes between leach and proposed ICA- LEACH

\section{CONCLUSION}

In wireless sensor networks, selecting appropriate cluster heads plays an important role in improving the network performance. In this paper, we use imperialist competitive algorithm to optimize the cluster heads selection by taking into account parameters such as the energy in LEACH algorithm. This protocol are based on the Leach algorithm. In this algorithm we conquered the shortcomings of Leach considering node's remained energy and its location which directly affect the energy consumption of the network with using imperialist competitive algorithm and improve the efficiency of the algorithm. The experimental results show that the proposed method causes more energy efficiency and as a result the network lifetime is increased.

In the future work, a possible improvement is simultaneous deployment of cluster heads and common nodes for better load balance of cluster heads when deploying nodes using other meta heuristic such as PSO and IWO algorithms. Other possible improvement is selection of mobile nodes as cluster heads and finds an optimal path to collect data from all clusters.

\section{REFRENCES}

[1] Akyildiz, I.F, Su, W. Sankarasubramaniam, Y ,Cayirci, E. 2002. "Wireless sensor networks: a survey. Computer networks", 38, 393-422.

[2] Wang H, Agoulmine N, Ma M, Jin YL. Network lifetime optimization in wireless sensor networks. IEEE J Sel Areas Commun 2010;28(7):1127-37

[3] Prabhu, S.B. Sophia, S. 2011. "A survey of adaptive distributed clustering algorithms for wireless Sensor networks". International Journal of Computer Science and Engineering Survey, 2,165-176.

[4] Heinzelman, W.B.; Chandrakasan, A.P.; Balakrishnan, H. 2002. "An application-specific protocol Architecture for wireless microsensor networks". Wireless Communications, IEEE Transactions, 1, 660-670.

[5] Loscrì V, Morabito G, Marano S. 2005. "A two-levels hierarchy for low-energy adaptive clustering hierarchy" (TL-LEACH). In: Proc. 62nd IEEE vehicular technology conference: VTC-2005-Fall,p 1809-13.

[6] Tabibzadeh M, Sarram M, Adibnia F.2009. "Hybrid routing protocol for prolonged network lifetime in large scale wireless sensor network". In: International conference on information and multimedia technology, Jeju Island, South Korea.

[7] Lindsey S, Raghavendra CS. 2002. "PEGASIS: powerefficient gathering in sensor information systems". In: Proc IEEE aerospace conference, vol. 3;. p.1125-30.

[8] Handy MJ, Haase M, Timmermann D. 2002." Low energy adaptive clustering hierarchy with deterministic cluster-head selection" . In: Proc. 4th IEEE conference on mobile and wireless communication networks; p.368-72.

[9] Ghanavati, .M, Gholamian M .R, Minaie , .B, Davoudi .M. 2011." an efficient cost function for Imperialist competitive algorithm to find best clusters", Journal of Theoretical and Applied Information Technology, Vol. 29, No. 1.

[10] Shahvandi L .K, teshnehlab M.,Haroonabadi A. 2011. "A Novel Clustering in Wireless Sensor Networks used by Imperialist Competitive Algorithm", International journal of advanced engineering sciences and technologies",Vol 29. No. 2,pp. $276-280$. 